\title{
"NEAR MAGIC ANGLE" SAMPLE SPINNING IN NMR SPECTRA OF MOLECULES ORIENTED IN INDIVIDUAL AND MIXED LIQUID CRYSTALS OF OPPOSITE DIAMAGNETIC ANISOTROPIES
}

\author{
B.S. ARUN KUMAR, N. SURYAPRAKASH, K.V. RAMANATHAN and C.L. KHETRAPAL ${ }^{1}$ \\ Indian Institute of Science, Bangalore 560012, India
}

Received 6 February 1987

\begin{abstract}
The dynamics of the liquid crystal director in "near magic angle" sample spinning has been investigated in mesophases with positive and negative diamagnetic susceptibility anisotropies and their mixtures with near-zero macroscopic diamagnetic anisotropy. To this end the deuteron quadrupolar splitting of deuterochloroform dissolved in liquid crystals has been monitored. In systems with weakly positive diamagnetic susceptibility anisotropy $(\Delta \chi)$, the director has been observed to switch from an orientation parallel to the spinning axis at low rotational speeds to one perpendicular to the spinning axis at high rotational speeds when the angle $(\theta)$ the axis of rotation makes with the magnetic field is smaller than the magic angle $\left(\theta_{\mathrm{m}}\right)$. For systems with a small negative $\Delta \chi$ similar director behaviour has been observed for $\theta$ greater than $\theta_{\mathrm{m}}$.
\end{abstract}

\section{Introduction}

The director of a nematic liquid crystal of a static sample orients either parallel or perpendicular to an external magnetic field, depending on the sign of the diamagnetic susceptibility anisotropy $(\Delta \chi)$. This property has given rise to a large number of interesting applications $[1,2]$ of NMR spectroscopy of oriented systems. The rather recent discovery that the director of a liquid crystal, when spun in a magnetic field at an angle other than $0^{\circ}$ or $90^{\circ}$ with respect to the magnetic field at a speed higher than a critical speed $\left(\omega_{c}\right)$, can be made to orient along or perpendicular to the spinning axis $[3,4]$ has opened up other interesting possibilities for applications [5,6]. The spectral pattern and the dynamics of the director orientation in a spinning liquid crystal sample has also attracted the attention of other groups [7-9]. In this paper, we present the results of NMR investigations carried out on a quadrupolar nucleus $\left({ }^{2} \mathrm{H}\right.$ in deuterated chloroform) in single as well as in mixtures of liquid crystals spinning at angles close to the magic angle. The liquid crystals used were trans-p-pentyl-4cyanophenyl)-cyclohexane (S-1114) with $\Delta \chi>0$ and

1 To whom correspondence should be addressed. a ternary eutectic mixture of propyl, pentyl and heptyl bicyclohexyl carbonitrile (ZLI-1167) with $\Delta \chi<0$. We have carried out these experiments at different spinning speeds and have observed that in mixtures of liquid crystals the director can switch orientation with the spinning speed when the axis of sample rotation makes an angle $\theta$ less than the magic angle $\left(\theta_{\mathrm{m}}\right)$ for systems with $\Delta \chi$ weakly positive and when $\theta$ is greater than $\theta_{\mathrm{m}}$ for systems with $\Delta \chi$ weakly negative.

\section{Experimental}

Deuteron NMR studies in $8 \mathrm{wt} \%$ solutions of deuterochloroform $\left(\mathrm{CDCl}_{3}\right)$ dissolved in $\mathrm{S}-1114$ and ZLI-1167 were undertaken. In addition, a 1:1 mixture of ZLI-1 167 and S-1 114 with about $8 \mathrm{wt} \% \mathrm{CDCl}_{3}$ was also prepared. Such a mixture has macroscopic $\Delta \chi \approx 0$ and has spectra corresponding to the two orientations of the liquid crystal director oriented along and orthogonal to the magnetic field [10]. To such a mixture, S-1114 or ZLI-1167 was added slightly in excess to make the macroscopic $\Delta \chi$ either weakly positive or negative respectively. The experiments were carried out on a Bruker MSL-300 FT-NMR 
spectrometer. The deuterium resonance frequency was $46.07 \mathrm{MHz}$. A double-bearing magic-angle sample spinning probe was employed. The spinning speeds could be varied from 200 to $1600 \mathrm{~Hz}$, but the variation of the angle of the sample rotation axis was restricted to $4-5^{\circ}$ on either side of the "magic angle".

\section{Results}

Figs. 1A and 1B show ${ }^{2} \mathrm{H}-\mathrm{NMR}$ spectra of $\mathrm{CDCl}_{3}$ in S-1114 spun at two different speeds, the angle $\theta$ the rotor axis makes with the magnetic field being $53^{\circ}\left(\theta<\theta_{\mathrm{m}}\right)$. The spectra for $\theta=56.5^{\circ}\left(\theta>\theta_{\mathrm{m}}\right)$ are shown in figs. $1 \mathrm{C}$ and $1 \mathrm{D}$. The spectrum of the static sample is also included in fig. 1 (E) for comparison. For $\theta<\theta_{\mathrm{m}}$, the director aligns parallel to the spinning axis [3] and the spectrum consists of a doublet with separation $598 \mathrm{~Hz}$ scaled by a factor $\Delta=$ $\frac{1}{2}\left(3 \cos ^{2} \theta-1\right)$ as compared to the static sample. For different spinning speeds, the spectra appear to be similar except for the position of the rather weak

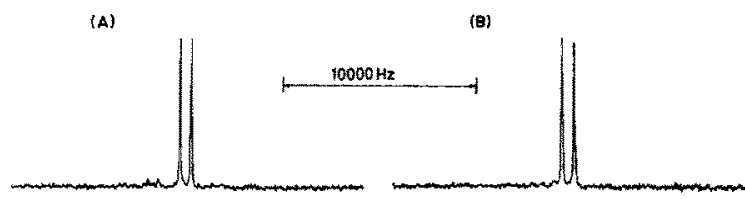

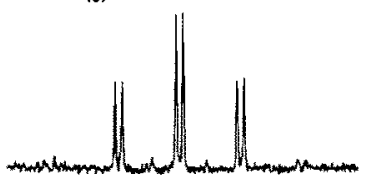

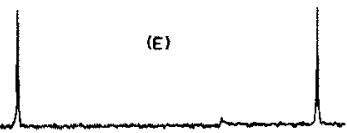

Fig. 1. ${ }^{2} \mathrm{H}-\mathrm{NMR}$ spectra of deuterochloroform oriented in the nematic phase of S-1114 for two different orientations $(\theta)$ of the axis of rotation with reference to the magnetic field and for two different spinning speeds (RO). DS refers to the doublet splitting. Solute concentration $=8$ wt $\%$,temperature $=292 \mathrm{~K}$, spectrometer frequency $=46.07 \mathrm{MHz}, \mathrm{Hz} /$ point $=12$. (A) $\theta=53^{\circ}$ $\left(<\theta_{m}\right), \mathrm{RO}=1660 \mathrm{~Hz}, \mathrm{DS}=598 \mathrm{~Hz}$; (B) $\theta=53^{\circ}\left(<\theta_{\mathrm{m}}\right)$, $\mathrm{RO}=1070 \mathrm{~Hz}, \mathrm{DS}=610 \mathrm{~Hz}$; (C) $\theta=56.5^{\circ}\left(>\theta_{\mathrm{m}}\right), \mathrm{RO}=1565$ $\mathrm{Hz}, \mathrm{DS}=354 \mathrm{~Hz}$; (D) $\theta=56.5^{\circ}\left(>\theta_{\mathrm{m}}\right), \mathrm{RO}=1060 \mathrm{~Hz}, \mathrm{DS}=366$ $\mathrm{Hz}$; (E) static sample. spinning side bands which appear due to a distribution in the director orientation around the spinning axis. For $\theta>\theta_{\mathrm{m}}$ the director is expected to orient in a plane perpendicular to the spinning axis [3]. Since the spinning speeds employed are smaller than the quadrupolar splitting ( $15405 \mathrm{~Hz}$ for the static sample: fig. 1E), the spectrum splits into side bands, each side band being a doublet with separation proportional to $-\frac{1}{2} \Delta[7]$. If the director is oriented exactly perpendicular to the spinning axis only even-order side bands are expected [3]. The appearance of weak odd-order side bands (fig. 1C) indicates that the director orients in other directions also. The oddorder side bands are more prominent at lower spinning speeds (fig. 1D). However the splitting of the centre band remains nearly the same at different spinning speeds and the lines remain sharp. This indicates that the director orientation has a distribution about the plane perpendicular to the spinning axis with fast exchange between different orientations. Although this distribution of the director orientation appears to be different at different spinning speeds, the average orientation of the director in all these cases is perpendicular to the spinning axis. At low spinning speeds (fig. ID), a differential intensity between the two lines of any side band except the centre band is observed. This can be understood as follows. The observed quadrupolar spectrum can be considered to be the superposition of a satellite line appearing at frequency $\frac{1}{4} \Delta q_{\mathrm{a}}$ from the centre and its sidebands $\left(q_{\mathrm{a}}\right.$ is the quadrupolar splitting when the director aligns parallel to the magnetic field) appearing at intervals of $\pm \omega_{\mathrm{r}}$ from it $\left(\omega_{\mathrm{r}}\right.$ is the sample rotation frequency) and another satellite line appearing at frequency $-\frac{1}{4} \Delta q_{\mathrm{a}}$ from the centre and its side bands. For orientations of the director other than $90^{\circ}$ with respect to the spinning axis, side bands corresponding to any one satellite appearing at $+n \omega_{r}$ and $-n \omega_{\mathrm{r}}$ can have different intensities. This will then result in doublets of unequal intensities for side bands of the same order $[10,11]$.

For $\mathrm{CDCl}_{3}$ dissolved in ZLI-1167 $(\Delta \chi<0)$, behaviour similar to that mentioned above is observed with the difference that for $\theta>\theta_{m}$ the director orients parallel to the spinning axis and for $\theta<\theta_{\mathrm{m}}$ the average orientation of the director is perpendicular to the spinning axis.

The effect of sample rotation on a mixture of liq- 

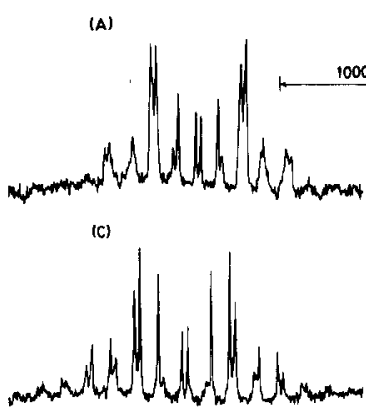

(B)
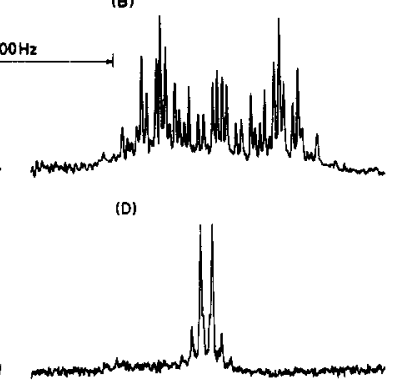

Fig. 2. ${ }^{2} \mathrm{H}-\mathrm{NMR}$ spectra of deuterochloroform in a "near" critical mixture of S-1114 and ZLI-1167 with a slight excess of S1114 such that the macroscopic diamagnetic susceptibility anisotropy $(\Delta \chi)$ is positive but close to zero. The orientations $(\theta)$ of the axis of rotation with reference to the magnetic field, the spinning speed (RO) and the doublet splitting (DS) are: (A) $\theta=56^{\circ}\left(>\theta_{\mathrm{m}}\right), \mathrm{RO}=1150 \mathrm{~Hz}, \mathrm{DS}=269 \mathrm{~Hz}$; (B) $\theta=56^{\circ}\left(>\theta_{\mathrm{m}}\right)$, $\mathrm{RO}=480 \mathrm{~Hz}, \mathrm{DS}=269 \mathrm{~Hz}$; (C) $\theta=53^{\circ}\left(<\theta_{\mathrm{m}}\right), \mathrm{RO}=1200 \mathrm{~Hz}$, $\mathrm{DS}=293 \mathrm{~Hz}$; (D) $\theta=53^{\circ}\left(<\theta_{\mathrm{m}}\right), \mathrm{RO}=470 \mathrm{~Hz}, \mathrm{DS}=598 \mathrm{~Hz}$. Other experimental conditions are the same as in fig. 1.

uid crystals with $\Delta \chi \approx 0$ has also been examined. It is observed that at all spinning speeds above the critical value, the director always aligns perpendicular to the spinning axis [9]. In the present study, the effect of specimen rotation on samples with $\Delta \chi$ small but not zero has been investigated. Thus, a "critical" mixture [12] of S-1114 and ZLI-1167 containing $\mathrm{CDCl}_{3}$ where the two spectra corresponding to two orientations of the liquid crystal director "coexist" in a static sample was prepared. From such a sample two sets of solutions - one in which S-1114 was slightly in excess and the other in which ZLI-1167 was slightly in excess - were prepared. The spectra of the sample with S-1114 in excess are shown in fig. 2 . As can be seen (traces A and B) for a given $\theta$ $\left(\theta>\theta_{m}\right)$, the changes observed for different spinning speeds occur only in the side band patterns and can be understood as being due to varying distribution patterns of the director orientation at different spinning speeds. For $\theta<\theta_{\mathrm{m}}$, the spectral patterns show significant changes when the spinning speed is varied. Fig. $2 \mathrm{C}$ corresponds to an orientation of the director perpendicular to the spinning axis, whereas fig. 2D corresponds to a parallel orientation. This can be seen from the presence of spinning side bands over a significantly large range of frequencies for rotor speed of $1200 \mathrm{~Hz}$. Further, the splitting of the central doublet in fig. $2 \mathrm{D}(598 \mathrm{~Hz})$ is nearly double that in fig. $2 \mathrm{C}(293 \mathrm{~Hz})$.

Thus for a single liquid crystal system such as S1114 with positive $\Delta \chi$, the director orientation remains parallel to the spinning axis (figs. 1A, 1B) at different spinning speeds above a critical value for $\theta<\theta_{\mathrm{m}}$. But for a system with $\Delta \chi$ nearly zero and positive, for $\theta<\theta_{\mathrm{m}}$, the director switches from an orientation along the spinning axis at low speeds to one perpendicular to it, at high speeds (figs. 2C, 2D).

We have observed a similar behaviour for the "critical" mixture with ZLI-1167 slightly in excess $(\Delta \chi<0)$ except that for $\theta<\theta_{\mathrm{m}}$, no change in director orientation is observed when the spinning speed changes, and for $\theta>\theta_{\mathrm{m}}$, the director switches orientation when spinning speed changes. In a "critical" mixture $(\Delta \chi \approx 0)$, away from the "critical" temperature $\Delta \chi$ is weakly positive or negative and it has been possible to observe a similar behaviour in the director orientations as a function of spinning speed.

A natural extension of these experiments is to investigate the director orientation behaviour in systems with larger $\Delta \chi$ such as single liquid crystal systems. Our experiments to observe switching of the director orientation in such a system have not been successful as it appears that the spinning speeds required for this to happen are beyond the experimental capabilities of our system.

\section{Conclusions}

The above experiments confirm that the director orientation depends on $\Delta \chi$, the spinning speed and the angle the spinning axis makes with the magnetic field. By suitably adjusting these three parameters, it is possible to orient the director either parallel or perpendicular to the axis of sample rotation.

\section{Acknowledgement}

We are grateful to Professor Anil Kumar and Dr. E.D. Becker for helpful discussions. 


\section{References}

[1] J.W. Emsley and J.C. Lindon, NMR spectroscopy using liquid crystal solvents (Pergamon Press, New York, 1975) pp. 1-367.

[2] C.L. Khetrapal and P. Diehl, NMR - Basic principles and progress, Vol. 1 (Springer, Berlin, 1969) pp. 1-95.

[3] J. Courtieu, D.W. Alderman, D.M. Grant and J.P. Bayles, J. Chem. Phys. 77 (1983) 723.

[4] R. Teeaar, M. Alla and E. Lippmaa, Org. Magn. Reson. 19 (1982) 134.

[5] C.L. Khetrapal, B.S. Arunkumar, K.V. Ramanathan and N. Suryaprakash, J. Magn. Reson., to be published.
[6] B.M. Fung and M. Gangoda, J. Chem. Phys. 83 (1985) 3285.

[7] T. Vaananen, J. Jokisaari and M. Selantaus, Chem. Phys. Letters 129 (1986) 399.

[8] J.P. Bayle, A. Khandar-Shahabad, J. Courtieu and S.K. Kan, Liq. Cryst. 1 (1986) 371.

[9] J.P. Bayle, A. Khandar-Shahabad and J. Courtieu, Liq. Cryst. 1 (1986) 189.

[10] B.S. Arunkumar, K.V. Ramanathan and C.L. Khetrapal, to be published.

[11] J.P. Bayle, A. Khandar-Shahabad, P. Gonord and J. Courtieu, J. Chim. Phys. 83 (1986) 177.

[12] C.L. Khetrapal and A.C. Kunwar, Chem. Phys. Letters 82 (1981) 170. 